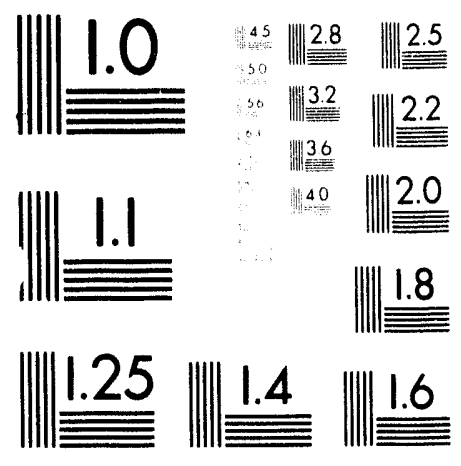



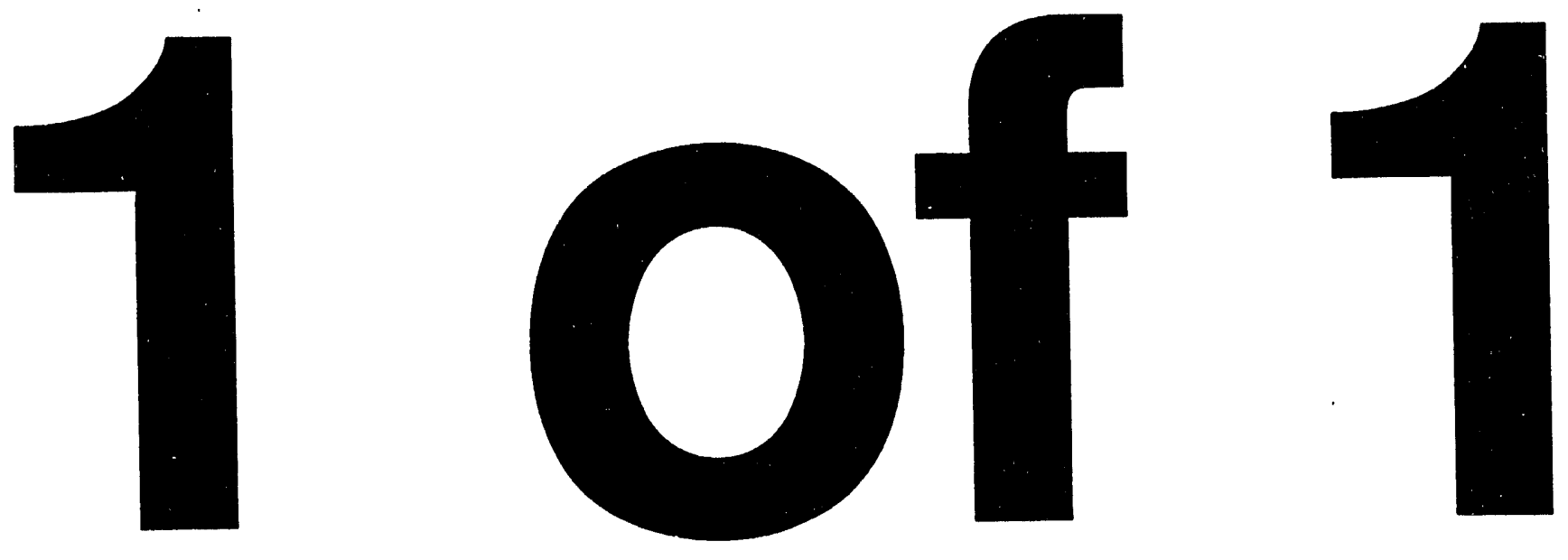
Distribution

Category UC-705

SAND 93-(1871

Unlimited Release

Printed November 1993

\title{
Modeling Data Throughput on Communication Networks
}

\author{
John M. Eldridge \\ Sandia National Laboratories \\ Division 1955 \\ Albuquerque, New Mexico 87185
}

\begin{abstract}
New challenges in high performance computing and communications are driving the need for fast, geographically distributed networks. Applications such as modeling physical phenomena, interactive visualization, large data set transfers, and distributed supercomputing require high performance networking |St 89|| $\mathrm{Ra} 92|| \mathrm{Ca} 92 \mid$. One measure of a communication network's performance is the time it takes to complete a task -- such as transferring a data file or displaying a graphics image on a remote monitor. Throughput, defined as the ratio of the number of useful data bits transmitted per the time required to transmit those bits, is a useful gauge of how well a communication system meets this performance measure. This paper develops and describes an analytical model of throughput. The model is a tool network designers can use to predict network throughput. It also provides insight into those parts of the network that act as a performance bottleneck.
\end{abstract}

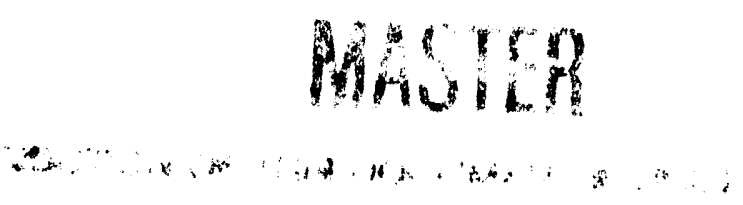




\section{Table of Contents}

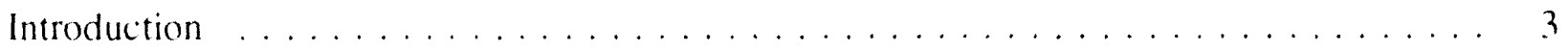

Model Description . . .............................

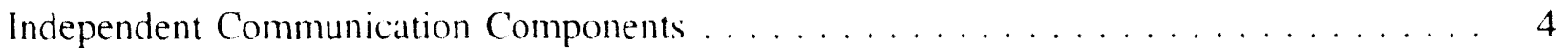

Process Sturtup ........................ 5

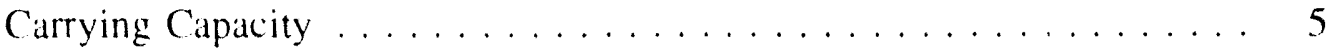

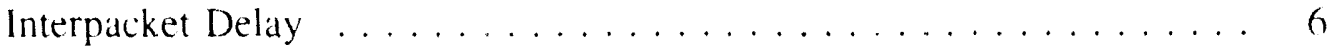

Protocol Waiting ...................... 7

Error Recovery . . . . . . . . . . . . . . . . . . . y

Parameter and Component Effects ..................... 10

Network Decomposition . . . . . . . . . . . . . . . . . . . . 12

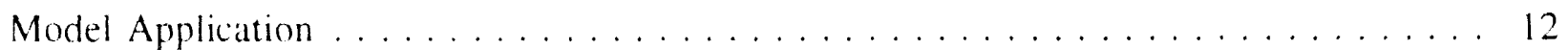

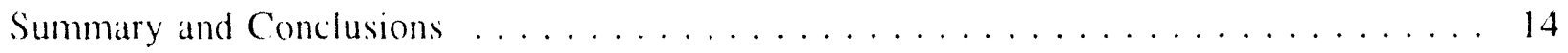

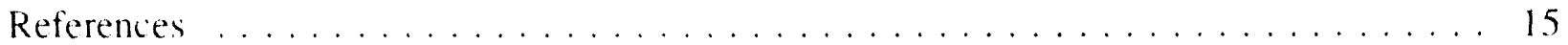




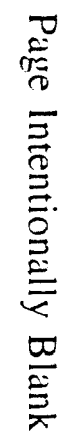




\section{Introduction}

New challenges in high performance computing and communications are driving the need for fast, geographically distributed networks. Applications such as modeling physical phenomena, interactive visualization, large data set transfers, and distributed supercompunng require high performance networking $\mid S t 89\left\|R_{4} 92\right\|\left(\mathrm{C}_{1} 92 \mid\right.$. One measure of a communication network's performance is the time it lakes to complete a task -- such as transferring a data file or displaying a graphics image on a remote monitor. Throughput, defined as the ratio of the number of useful data bits transmitted per the time required to transmit those bits, is a useful gauge of how well a communication system meets this performance measure.

Current Local Area Network technologies including Ethernet and Fiber Distributed Data Interface $\mid$ Th $89 \mid$ operate with transmission line rates of $1(1)-10(1)$ million bits per second (Mbps). Higher transmission line rates and switching rates, as offered by technologies such as SONET $\mid \mathrm{Ba} X 9)$ and Asychronous Transfer Mode [Go92||Ly92|, will be required for applications $\left.\mid R_{a}\right)_{2}||\left(a^{(9)} 2 \mid\right.$ that need (iiga bit per second throughput. However, increased transmission line rates alone may not necessarily lead to throughput increases. Network performance is dependant on many variables -- the computers involved, the data communication medium and line rate, and the communication protocol used. To achieve higher throughput requires higher performance from all parts of the network; not just the transmission medium. This paper develops and describes an analytical model of throughput. The model is a tool network designers can use to predict network throughput. It also provides insight into those parts of the network that act as a performance bottleneck.

\section{Model Description}

A pratctical data communication system minimally contains at computer transmitting datat, a computer receiving data, a communication medium, and the communication protocol. The computer originating a data transmission is called the "Sender," and the computer accepting or receiving the data is called the "Receiver." The Open Systems Interconnection - Basic

Reference Model |ISOS4l describes computer communications in terms of layers or levels where each layer builds upon its predecessor. Each layer presents certain services to higher layers and shields the higher layers from the implementation details of the lower layers. Depending on the layer at which throughput is measured, different throughput values may be observed. At each layer, the definition of useful data may be different since there may be different tasks that contribute to the total transmission time. For instance, application layer throughput may include the time to read and write data to or from disk storage while transport or physical layer throughput does not include time for this task. The throughput at the application layer and transport layer will be different.

Throughput by definition is the ratio of the number of useful data bits transmitted per total time of transmission, and it has units of bits per second. If a computer sending data transmits $B$ useful data bits in $t_{1}$ seconds, then the throughput of the transmission is $T_{1}$, bits per second as shown in |Equ. |A|. Several transmission process components contribute to the total time needed to complete the transmission. Processes that significantly add to the total transmission time include: communication process startup, protocol and data processing on the Sender and Receiver, transmission media clocking rate, congestion control, and error detection and recovery. 
If process components are independent of one another, so that they occur sequentially in time, then the total transmission time is the sum of the times required to complete each process component. The time required to complete each independent process component does not overlap the time required to complete another independent process component. In other words, independent process components are orthogonal to each other. Specific independent processes are described later. For a data transmission that has a number, $k$, of independent process components, the total transmission time is the sum of the time required for each of the $k$ process components as shown in |Equ. 1B|. An arbitrary or $i^{\text {th }}$ process component for a data transfer takes some amount of time $t_{1}$ to complete, and it contributes to the total transmission time. If one considers independent processes individually, then each independent process can be thought of as proceeding at its own throughput. Here, the process throughput is the

$$
\begin{aligned}
& \mid \text { Equ.1A| } T_{P}=\frac{B}{t_{T}} \\
& \mid \text { Equ. }|B| \quad t_{i}=\sum_{i=1}^{k} t_{1} \\
& \text { |Equ. 1C| } \frac{1}{T_{p}}=\sum_{i=1}^{k} \frac{1}{T_{m}} \\
& T_{p}=\text { total throughput } \\
& B=\text { useful bits transmitted } \\
& t_{1}=\text { total transmission time } \\
& t_{1}=\text { transmission time for } i^{\text {th }} \\
& \text { independent process } \\
& k=\text { number of independent processes } \\
& T_{I^{\prime \prime}}=\text { throughput of } i^{\text {th }} \\
& \text { independe'nt processis }
\end{aligned}
$$

ratio of total useful data transmitted by the time to

complete that particular process component. Total throughput can be determined from the process throughputs as shown by $\mid$ Equ. $1 \mathrm{Cl}$.

Analytical equations describing the component throughputs are developed for the case where a point-to-point. full-duplex data circuits directly connects the Sender and Receiver. Since the Sender and Receiver are not directly connected in many networks, a method for removing this restriction is described later. The data circuit has a set of describing characteristics. It operates at some fixed line speed $L$. That is the energy pulse of a data bit emitted onto the circuit has a duration of $1 / \mathrm{L}$, seconds. The data circuit also exhibits latency due to the speed of electromagnetic propagation over the circuit's length and due to propagation and queving delays through data circuit hardware. A data bit's energy pulse injected into one end of the circuit will arrive at the other end after a delay of $d_{1}$ seconds due to latency. Unusual event. may cause the bit's energy pulse to be drastically distorted as it propagates along the length of the circuit. In this case, a bit errors occurs. Thus, the circuit has a bit error rate.

\section{Independent Communication Components}

The basic throughput model described in |Equ. 1/ can be applied to any data communication protocol. However, the specific values of the independent process components would change from one protocol to the next depending on the protocol specifics. Some process components may exist for one protocol but may not exist for another. Discussion of the model is focused by using the Transmission Control Protocol/Internet Protocol (TCP/IP) |Co91| as a basis to describe and develop the model of this paper. However, the development would be similar for other protocols. 
Multiple sets of independent processes may exist for a given network and communication session. However, the resultant total throughput resuits for each set will be the same. Some sets may be more useful for network analysis and design than other sets. One independent process set for TCP/IP includes communication process startup time, network carrying capacity, computer processing time, protocol acknowledgement, and error recovery. This set corresponds to distinct functional pieces of the network.

Procesis Startup -- When a user requests a data transfer between two computers, the request initiates execution of communication tasks on the two computers, and a transmission protocol "handshake" occurs. The handshake allows the two computers to negotiate and use protocol options and settings that are common to both. The transport level protocol will usually exchange information to establish a virtual connection between tasks and to adjust the transmit and receive buffers sizes for the session. For cases where the transmit throughput capacity of the Sender is greater than the receive throughput capacity of the Receiver, the Sender and Receiver will need to negotiate and adjust flow control parameters. Process startup time, $t_{s p}$, on different computers may vary considerably depending on system resources, system load, or system capability. Protocol handshake is sensitive to the propagation delay between the Sender and Receiver because delay increases the time required to convey status between the computers. Protocol handshake contributes to the startup time. After the handshake is completed and the Sender begins to transmit user data, the data will not arrive at the Receiver until after a time equal to the data circuit's propagation delay. This initial transit time also adds to the process startup time.

The process startup time occurs at the beginning of the data transfer, and it is independent of the amount of user data sent. Process startup time is at least as long as the propagation delay because of the initial data transit delay between Sender and Receiver. In cases where a small amount of user data is transmitted per session. the process startup time may be a significant component of the total time required for data transmission. In cases where a large amount of data is transmitted, for example the transfer of a large data file, the process startup time is small compared to the total transmission time and it can usually be ignored in throughput calculations. Process startup and flow control adjustment component throughput is

$$
T_{w}=\frac{B}{T_{S H}}
$$

Carrying Capacity -. Carrying capacity is the throughput of a communication circuit considering the effects of protocol overhead and network loading. Think of carrying capacity as that part of a data circuit's bandwidth allocated to transferring user data. The effects of protocol overhead, network loading, and multiple network segments factor into the determination of carrying capacity. User data sent from the Sender to the Receiver is broken into protocol data units and transferred as packets. The Receiver processes the data packets and then sends an acknowledgement message back to the Sender indicating it received the data without errors. Each data packet contains both user data, $\mathrm{P}_{\mathrm{D}}$, bits, and "overhead" data, $P_{0}$, bits. The protocol uses the overhead data for packet addressing, packet routing, signalling, and error detection. Therefore, to the user only part of the bandwidth of the data circuit is available. Carrying capacity is the throughput of the transmission medium dedicated to 
transmitting user data. It takes into consideration the extra time required to transmit overhead data and data other than user data.

Each packet transmitted is $P_{S}\left(=P_{1},+P_{1}\right)$ bits long. The time required to emit one packet onto the circuit neglecting media sharing is $P_{S} / L_{s}$. Carrying capacity, $T_{1}$, is then the total number bits transmitied divided by the component's transmission time. For the Sender to move completely a data block of B bits to the Receiver, the Sender must transmit an integral number of packets equal to the smallest integer greater than $\mathrm{B} / \mathrm{P}_{1}$. The total transmission time due to carrying capacity is the product of the number of packets transmitted and the time to transmit a single packet.

$$
T_{r r}=\frac{P_{l} L_{r}}{P_{s}} .
$$

Interpacket Delay - Each time the Sender transmits a packet, it must retrieve and format the data into a packet as defined by the communication protocol. It then emits the packet out of the physical interface to the data circuit. The Sender has limits on how yuickly it can perform these tasks. Therefore, there is a time delay between when the Sender can transmit the last bit of one packet and the first bit of the next packet. The time delay between packets, or the interpacket delay, $t_{p^{\prime}}$, relates to the Sender's internal throughput and processing capabilities.

Computers are not infinitely fast. The CPU, memory, and bus operating speeds limit how quickly the computer can execute the protocol and move data. The protocol coding efficiency, the operating system, and system load further limit how quickly the Sender processes packets. Various applications require different system resources which perform with different capabilities. For instance, retrieving data from memory is quicker than retrieving it from disk. Multi-tasking systems further spread system resources over multiple applications.

The internal throughput and processing capabilities of the Sender affect the interpacket delay. Interpacket delay increases with increasing packet size because of limited memory, bus, and storage device transfer speeds. Regardless of packet size, a minimum amount of processing is required to generate the routing, addressing, and error detection data (i.e., the "overhead" data) for each packet. Clark $|C| X Y \mid$ has investigated the processing needs for TCP/IP. His work indicates that the Sender's memory bandwidth and operating system are the largest contributors to interpacket delay.

As a first approximation, interpacket delay is linear with respect to packet size. Because of variances between computer systems, interpacket delay is best determined by empirical measurement. Measurements of interpacket delay for scientific workstations typically range from about $(1.3 \mathrm{mS}$ for a 512 byte packet to about $2.5 \mathrm{mS}$ for a $430(1)$ byte packet /Ch92/. These interpacket delay values are for data transfers from memory. Data transfers from disk storage result in larger interpacket delays.

For the Sender to transmit B bits to the Receiver, interpacket delay contributes to the total transmission time. The product of the per packet delay, $t_{p}$, and the total number of packets 
transmitted is equal to the time contributed. Thus, the throughput component due to interpacket delay, $\mathrm{T}_{\mathrm{P} \mid \mathrm{l}}$, is

$$
\begin{aligned}
& T_{P(l)}=\frac{P_{1}}{t_{P(l)}}, \text { where } \\
& \left.t_{P(l)}=\left(\text { measured (onstant }{ }_{1}\right) P_{s}+\text { (measured constant } t_{2}\right) .
\end{aligned}
$$

Protocol Waiting - - The Transmission Control Protocol (TCP) ensures error free data delivery, and the TCP's flow control mechanism limits the Sender's data transmission rate if the communications circuit and Receiver cannot accept data as quickly as the Sender can deliver the data. A technique known as positive acknowledgement with retransmission accomplishes error free data delivery. The Sender's TCP packages data into packets to send to the Receiver. The Receiver returns to the Sender an acknowledgement message indicating that it correctly received the packet. The Sender retransmits packets that are not acknowledged as received correctly. This acknowledgement mechanism may induce circuit idle time if the Sender must stop transmitting and wait for a returned acknowledgement before sending the next packet. This wait period increases as the propagation delay between Sender and Receiver increases.

TCP uses a sliding window technique for packet acknowledgement and flow control. This technique allows the Sender to transmit multiple packets before it receives an acknowledgement. The window size, $W_{\text {, determines the number of outstanding }}$ unacknowledged packets. The window size is the maximum amount of user data that unacknowledged packets may contain. If the window size is sufficiently large, then the Sender will receive the acknowledgements for the first packets it transmitted before it transmits the last packet of the window. Otherwise the Sender completes transmission of the window before receiving acknowledgements to the first packets. For this second case, the Sender is idle until it receives returned acknowledgements.

Assume a wait for acknowledgement idle time of 1 seconds occurs after the Sender transmits each block of user data equal to the window size, $W$. The delay may in fact be zero if the time to transmit the window is sufficiently large so that the Sender receives packet acknowledgements before it exceeds the transmission window size. The time that the Sender is idle after each window transmission, is the difference between the round trip time and the window transmission time provided the difference is greater than zero. The round trip time, RTT, is the elapsed time between when the Sender sends a packet and when the Sender receives the acknowledgement for that packet. Increases in the propagation delay between Sender and Receiver proportionally increases the round trip time. The window transmission time. $W_{1}$, is the time the Sender takes to transmit the maximum allowed number of unacknowledged packets. Figure I shows the relationship between idle time, round trip time, and window time.

Several parameters including propagation delay, line speed, and the Sender's and Receiver's processing capabilities affect round trip time. When the Sender transmits a packet, the first bit of that packet reaches the Receiver after a time equal to the propagation delay $d_{1}$. The Receiver then moves the packet off the data circuit and into its receive buffers at the rate of 
the data circuit. Depending on the protocol implementation, the Receiver may not immediately acknowledge the packet to reduce network traffic. It may wait until it has received an integral number, $N$, of packets before returning an acknowledgement. TCP implementations commonly set the value of $\mathrm{N}$ to a third of the packets within a window. The interpacket delay, $t_{p^{\prime},}$, due to Sender protocol processing occurs between each

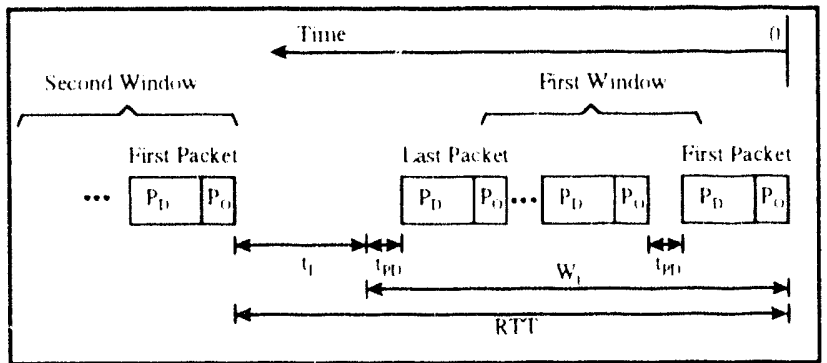

Figure 1 Relationship between wait for acknowledgement idle time, round trip time, and window time.

received packet. After the Receiver receives the $\mathrm{N}$ packets, it generates an acknowledgement message, $A_{s}$ bits long, and returns it to the Sender. Because of Receiver processing to generate an acknowledgement message, the Receiver returns the acknowledgement after a delay of $t_{R P}$, seconds. Thus, the round trip time is

$$
R T T=2 d_{l}+\frac{A_{S}}{L_{S}}+t_{R P}+N\left(t_{P l}+\frac{P_{S}}{L_{S}}\right) .
$$

The window transmission time is equal to the time required to transmit a packet multiplied by the number of packets contained in the window. The Sender emits the bits of each packet it transmits onto the data circuit at the line speed of the circuit. Again there is a interpacket delay between successively transmitted packets. The number of packets sent is then equal on the smallest integer greater than the value of the window size divided by the user data contained in a packet. The window transmission time is then

$$
H_{1}=\frac{H_{1}}{P_{1}}\left(t_{P 1}+\frac{P_{11}}{L_{n}}\right)
$$

The packet acknowledgement idle time after the transmission of the packets within a window is the difference between the round trip time and the window transmission time, and it is

$$
\begin{aligned}
& t_{l}=2 d_{l}+\frac{A_{S}}{L_{S}}+t_{R P}+\left(N-\frac{W_{S}}{P_{l}}\right)\left(t_{P l}+\frac{P_{S}}{L_{S}}\right) \\
& \text { Idle Time Per Window }=\left[\begin{array}{lll}
t_{1} & \text { if } \left.t_{P}>0\right) \\
0, & \text { if } t_{1} \leq()
\end{array}\right] \text {. }
\end{aligned}
$$

The total idle time while waiting for packet acknowledgements for the complete transmission is then the sum of all the idle periods between the transmission of packets of successive windows. The number of idle periods for the full transmission is $B / W_{s}$. If the idle time per window, $t_{1}$, is greater than zero, then throughput of the protocol waiting component $T_{P, W}$ is

$$
T_{P W}=\frac{W_{s}}{2 d_{L}+\frac{A_{S}}{L_{S}}+t_{r p}+\left(N-\frac{W_{s}}{P_{D}}\right)\left(t_{P D}+\frac{P_{s}}{L_{S}}\right)} .
$$


The processing capability of the Receiver, as well as the Sender, has limits. If the Receiver can accept data as quickly as the Sender can transmit it, then the Receiver will not lessen throughput. However, for the case where the Sender, Network, and Protocol combination can deliver data more quickly than the Receiver can accept it. the Receiver must notify the Sender to transmit more slowly. The Sender accomplishes this by reducing the window size (after renegotiation with the Receiver). Thus, protocol waiting idle time increases. For circuits with small propagation delay the window may contain only a single packet.

Emor Recolery -- Error conditions during data transfer require the Sender and Receiver to handle an exception condition. Errors may occur due to noise on the data circuit, buffer overflows, or other events. When an error occurs, the Sender requires some amount of time to handle the error. This time for error processing is in addition to the time needed to transmit the data successfully. Though the periods required for error processing occur throughout the data transfer process, for the model the time for all error processing can be treated as a lump sum at the beginning or end of the process. The exact method the Sender and Receiver use to process an error condition will vary between protocols and protocol implementations. However, in general the transport layer of a reliable protocol on the Sender expects the Receiver to acknowledge correctly received data or data packets. The Sender must retransmit a lost or corrupted packet until it receives an acknowledgement for the packet.

Consider TCP for the purposes of model development. When the Sender transmits a packet. it will start a timer and wait for an acknowledgement. If the timer expires before the Sender's TCP receives the packet's acknowledgement, TCP will assume that the packet was lost or corrupted and retransmit it. TCP actually acknowledges the last correctly received byte of user data rather than the packet itself. This distinction however does not greatly affect the mocic! if one assumes that parkets ale acknowledged instead of the data stream. TCP uses a "Go-Back N" retransmission schence. This means that the Sender must remansmit all packets it transmitted after an unacknowledged packet even if the Receiver correctly received the later patkets.

When a packet's acknowledgement does not return to the Sender before the packet's retransmission timer expires, the Sender begins retransmitting starting with the unacknowledged packet. The state of the transfer returns to the same condition as if the Sender had never transmitted the unacknowledged packet. Therefore, whenever the Sender needs to retransmit an unacknowledged packet the error processing time for that packet is equal to the timer expiration period. The timer expiration period must be at least as long as the round trip time discussed above. Otherwise, the Sender would retransmit packets before acknowledgements could return. In practice, round trip time varies. To accommodate this variance the timer expiration period is a factor beta, $\beta$, larger than the round trip time. The Sender may need to retransmit a packet several times if retransmitted packets are also corrupted. If a packet has a probability $F$ of the Sender not receiving the acknowledgement for the packet, then one can calculate the expected number of retransmissions $E_{\mathrm{F}}$ for that packet. The independent throughput for error recovery is then 


$$
\begin{gathered}
T_{t: R}=\frac{P_{l}}{E_{P} \beta\left|2 d_{L}+\frac{A_{S}}{L_{S}}+t_{R r}+N\left(t_{P l)}+\frac{P_{S}}{L_{S}}\right)\right|} \text {, where } \\
E_{r}=\frac{F^{2}}{1-F} .
\end{gathered}
$$

The Sender will not receive a packet acknowledgement and will need to retransmit the packet if either the packet or the acknowledgement is lost. The effect of lost acknowledgements is not as great as the effect of lost data packets with TCP. Since TCP acknowledges the last correctly received byte of user data with each acknowledgement, it essentially reacknowledges all previously acknowledged packets. If an acknowledgment is lost, then the following acknowledgment performs the function of the lost acknowledgement. Thus, the probability of a packet error is about equal to the probability of the Sender not receiving an acknowledgement for the packet. Assuming a data circuits with Gaussian distributed bit errors, the probability of a packet error depends on the packet size in bits and the bit error rate of the circuit. Each bit transmitted over the data circuit has an error probability of ER. The more bits a packet contains then the higher the probability that the packet will be corrupted in transmission. For a data circuit with Gaussian distributed errors, the probability of a packet error is $F_{p}$, and it is approximately equal to $F$ if lost acknowledgements are ignored. $F_{1}$ is $|M a 78|$

$$
F=F_{1}=1-|1-E R|^{\prime}
$$

\section{Parameter and Component Effects}

Total network throughput is a function of all the independent process component throughputs. Depending on the operating parameters of the network, the effect of one process component's throughput may dominate total throughput. Total throughput improvement requires throughput improvement of the dominate process component. As described above, each independent component throughput depends on certain network operating parameters like packet size, line speed, propagation delay, or windww size. Throughput can be improved by network turing or by altering these parameters. Some of these parameters can be easily changed in software. Changes in others may not be possible or may require basic network design changes.

Parameter changes have differing affects on the process components as shown in Figure 2. This figure shows component throughput and total throughput as a function of packet size, line speed and propagation delay. In each plot, only the parameter of interest varies; all other parameters remain constant. As shown in the figure, the different component throughputs control total network throughput to varying degrees as the parameter values change. (One component process may be the dominate influence on total throughput for some value of a parameter, like line speed. However, if the value of the parameter is changed, another component process may become dominate. 


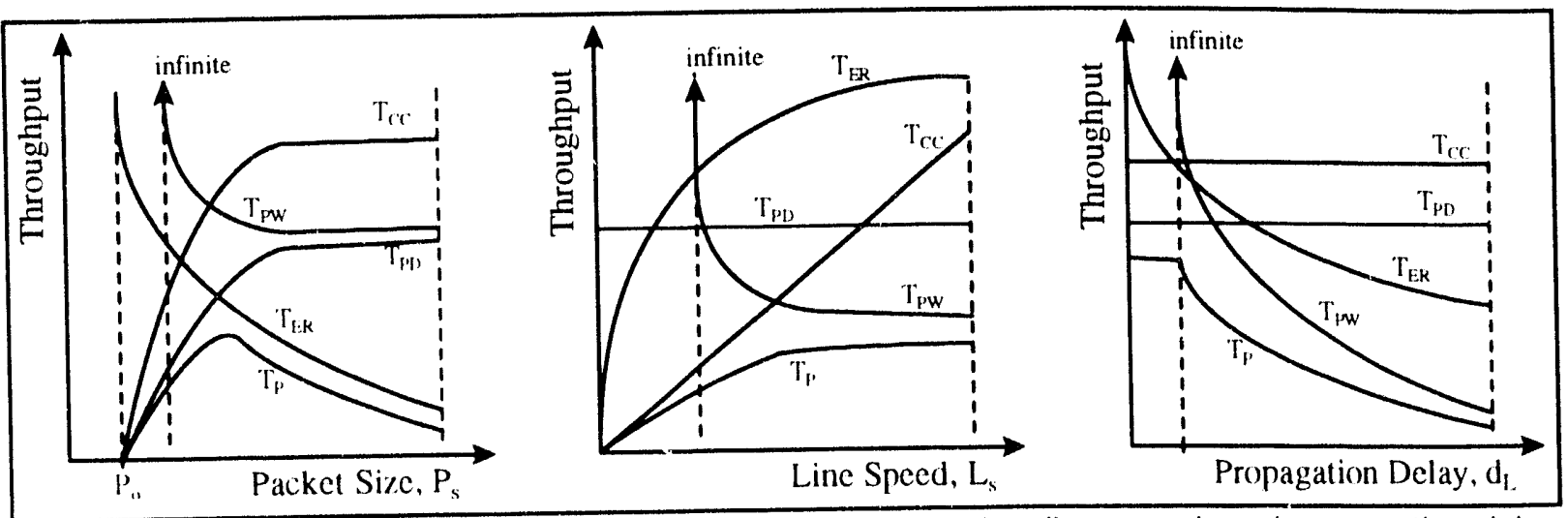

Figure 2 Throughput as a function of the parameters packet size, line speed, and propagation delay. Note: throughput plots are not to scale.

The interpacket delay component. $T_{p(1)}$, due to Sender processing is largely constant with respect to network parameters. However, $T_{[, I)}$ is sensitive to packet size for small packets due to the relatively larger amount of CPU processing to send data in small packets. Packet size affects carrying capacity when packets are small because of overhead data. Carrying capacity is insensitive to packet size for larger sized packets. With small packets, transmission of packet overhead contributes substantial time to the component. With large packets where packet overhead is insignificant, line speed limits carrying capacity. The error recovery component $T_{t: k}$ is sensitive to most network parameters. Any parameter that increases the probability of retransmission or the round trip time will decrease $T_{1: R}$. Packet size and data circuit error rate control the probability of retransmission. Large packet size and propagation delay increases round trip time while higher line speed reduces it. Most parameters also affect protocol waiting throughput, $T_{1, w}$. Window size and propagation delay predominantly determine whether or not $T_{p w}$ is finite.

For sufficiently large values of window size, protocol waiting idle time becomes zero. The window size value where idle time just becomes zero is optimum. Increasing window size beyond this value consumes computer resources (memory buffers) without improving throughput. Values of window size larger than the optimum may increase round trip time for those TCP/IP implementations that hold off returning acknowledgements based on window size. Optimum window size, $\mathrm{W}_{\mathrm{cpp}}$ is found by setting idle time to zero and solving for $\mathrm{W}_{\mathrm{s}}$. Its value is

$$
W_{i p t}=\frac{3 P_{D}\left(2 d_{L}+\frac{A_{s}}{L_{s}}+t_{k P}\right)}{2\left(t_{P D}+\frac{P_{S}}{L_{S}}\right)} .
$$

Total throughput cannot be optimized by individually optimizing the independent component throughputs. Maximizing one component may adversely affect another because of parameter interaction between components. Even in cases where parameter changes can be made to maximize one component without influencing another, individually optimizing that component may require excessive network resources without improving total throughput. 


\section{Network Decomposition}

The model equations developed above for each process component assumed a simple communication network with computers directly interconnected by a point-to-point data circuit. While this assumption eased development of the model, many data networks do not strictly meet this assumption. Commonly the network path between Sender and Receiver is at combination of network segments interconnected by routers and gateways. Each network segment may use a different network technology so that its line speed, error rate, and propagation delay may be different from those of other segments. To the Sender and Reciver, however, the network appears to present characteristics of line speed, error profile, and propagation delay equivalent to some point-to-point circuit. This is a "black box" view of the network. These characteristic parameters are random variables that will vary over time because of traffic loading effects on the network. If these parameters are statistically stationary for the duration of a data transfer, then this view holds and the network can be modeled as an equivalent point-to-point circuit.

Decomposing the network into an equivalent point-to-point data circuit requires the detemination of the network's carrying capacity. The line speed, packet overhead, and loading of each segment are different - thus, the segments" carry capacities are different. Data flows from the Sender to the Receiver througl, each network segment. The segment with smallest carrying capacity limits the data flow, and its capacity is equivalent to network's carrying capacity.

Each network segment also adds propagation delay and causes packet errors. Network propagation delay, is the sum of the propagation delay's of all segments. The network probability of an packet error is the combined probability that the packet contains an error from any network segment. The number of errors per packet is not significant, but what is significant is the probability that the packet has any error.

\section{Model Application}

To illustrate an application of the model, it is applied to the Wide Area Network of Figure 3. The Sender and Receiver connect to two separate Fiber Distributed Data Interface (FDDI) rings. Attached routers and a DS3 (44.7 Mbps). long haul circuit interconnect the rings. A DS3 delay and error simulator emulated long distance circuit effects by introducing propagation delay and line errors. The Sender is a Digital Equipment Corporation DEC Station $5(1)(1 / 20)()$, and the Receiver is a CRAY YMP 8/864. The Sender and Receiver use the Transmission Control Protocol and Internet Protocol. The network was built in a prototype test bed at Sandia National Laboratories, and throughput performance measurements were made on the network |Ti92\|Ch92]. Sandia National Laboratories is using this test bed for network performance measurements of remote supercomputing applications between its California and New Mexico sites.

Since one-way propagation delay, $\mathrm{d}_{1}$, between Sender and Receiver in a wide area network can significantly affect network performance [To92], throughput measurements are made for several values of propagation delay. Other parameter values remain constant. Throughput for this application is defined at the interface to the transport layer. The socket-to-socket metric code TTCP was used for making the measurements. TTCP is a small utility that performs 
memory to memory transfers between the Sender and Receiver via TCP/IP.

For large data transfers, the process startup time is small when compared to the total transmission time, and it may be neglected. In other words the throughput of the process startup component, $\mathrm{T}_{\mathrm{PS}}$, is much larger than the total transfer throughput $T_{1}$. All network throughput measurements satisfied this criterion, therefore the model illustration does not include $T_{\mathrm{ps}}$. One effect of excluding $T_{p}$, from the model is that $T_{1}$ is independent of the amount of data transmitted. Total throughput in terms of the independent process components is then

$$
\frac{1}{T_{r}}=\frac{1}{T_{(r)}}+\frac{1}{T_{P l)}}+\frac{1}{T_{P W}}+\frac{1}{T_{r i}} .
$$

For this example illustration, the TCP window size, $W_{s}$, and the user data payload, $P_{1}$, are set to 6.3 Kbytes and 4286 bytes respectively. This is the maximum window size that the Sender allows. TCP/IP generates 48 bytes of overhead. Link layeer overhead varies between the network segments. From network measurements, the interpacket delay is $1.17 \mathrm{mS}$.

The first step to apply the model is to decompose the network into its equivalent circuit as shown in Figure 3. This network has five distinct segments each with a distinct carrying capacity. The five segments include two FDDI rings, two routers, and 11/1: Delay and limen Sinulates

Figure 3 Test network's configuration and its equivalent.

Table I Test Network Parameter Values

$$
\begin{array}{ll}
W_{S}=63 \text { Kbytes } & t_{p D}=1.17 \mathrm{mS} \\
P_{0}=34.288 \text { bits } & t_{p H}=0.2 \mathrm{mS} \\
E R=10^{9} \text { errors per bit } &
\end{array}
$$$$
L_{s ;}=37.77 \text { Mbps, effective line speed }
$$
the DS3 circuit. The carrying capacities of the FDDI and DS3 segments were estimated from line speed, packet size and packet overhead. The router's carrying capacity was measured and found to have the smallest value for any segment. Therefore, the network's carrying capacity is equal to that of the routers. The error rate for the DS3 segment was set to $10^{-9}$ errors per bit. This line error rate is typical for a wide area data circuit, and rates of this size have been measured on data circuits between Sandia National Laboratories' California and New Mexico sites. The error rates for 
the other segments were ignored. Altering the setting of the DS3 delay simulator varied the total propagation delay. Throughput was then measured and calculated for each value of delay. The calculated values of throughput are close to the measured values. Results are given in Table I. When propagation delay is close to zero, the network carrying capacity, $\mathrm{T}_{(1,}$, and the Sender processing throughput, $\mathrm{T}_{\mathrm{P}, \mathrm{l})}$, dominate network throughput. Network carrying capacity and Sender processing throughput are respectively $37.2 \mathrm{Mbps}$ and 29.3 Mbps. As propagation delay increases, however, the wait for acknowledgement idle time increases. The protocol wating component's throughput, $T_{1}$, decreases causing overall throughput to decrease. Based on the window size used for this network, the model shows that $T_{1}$ is finite for propagation delay values greater than $10.7 \mathrm{mS}$, and it is infinite for smaller values of propagation delay. This result matches the measurements. The error rate for this network is relatively small. Thus, the error processing throughput, $T_{\text {I:R }}$ is relatively large at $2.3 \times 10$ ? Mhps compared to the other components's throughputs. Error processing with the line error rate used for this network application has little affect on network throughput.

Network throughput is about 9.8 Mbps with a propagation delay of $21 \mathrm{mS}$ which is the approximate propagation delay of a DS3 circuit between California and New Mexico. For this value of delay, the optimum window size is a little larger than 120 Kbytes. Setting window size to its optimum value would result in a network throughput of about 16.4 Mhps. Error processing throughput does not become significant until the probahility of a packet error, $F$, is near (1)(1). This packet error probability would correspond to a data circuit error rate of about one error in ten million bits, $E R=10^{\prime}$ errors per bit, assuming (ialussian distributed errors.

The Receiver was able to aceept data as quickly as the Sender and network could deliver it. Therefore, the Receiver did not need 10 limit throughput. Had the Receiver not been able to sustain the throughput of the Sender and network, the Receiver and Sender would have had to impose flow control restrictions by dynamically reducing the window size to increase the wait for acknowledgement idle time. A different window size value, up to the maximum allowed, woukd be negotiated for the different values of network propagation delay to limit throughput to match the Receivers capability.

\section{Summary and Conclusions}

This paper has presented a data throughput model that network analyst can use to predict network throughput. The model works for a variety of network configurations and operating conditions. The model also provides network analyst information on which processes control or limit throughput. Model development builds on the observation that different communication processes contribute to the total time required to complete a data transfer and that these processes occur sequentially. Thus, network throughput can be represented by a combination of throughputs attributed to the different communication processes or components.

The model application shows that user perceived throughput is less than the line speeds, or clocking rates, of the network's transmission media. While transmission media line speed places an upper limit on throughput, throughput depends on several independent processes components. 


\section{References}

$\mid$ Bary9| R. Ballart and Y.C. Ching, "SONET: Now it's the standard optical network, "IEEE Communications Magazine, pp. 8.15, March 1989.

$|C a y 2|$ C. E. Catlett, "In Search of Gigabit Applications," IEEE Communications Magazine, vol..30, no. 4, pp. 42-51. April 19\%2.

|C|X9| D. D. Clark, V. Jacohson, J. Romkey, H. Salwen, "An Analysis of TCP Processing Overhead", IEEE Communications Magazine, vol.27, no. 6.pp. 23-29, June 1989.

|Ch92| H. Y. Chen, J. A. Hutchins, N. Testi, "TCP Perfornance Analysis for Wide Area Networks," Supercomputing ' $\mathrm{H} 2$ Conference.

|Co91| Douglas E. Comer, "Internetworking with TCP/IP". Second Edition Volume I, Prentice-Hall. Engleword Clifts, 199l.

|Goy2] Steven A. Gossage. "Delivery of Very High Bandwidth With ATM Switches and SONET." Sandia National Laboratorics Report. SANI) $2-1295$, Octoher 1992.

ISSO84| ISO 7498. "Infonmation Processing Systems - Open System Interconnection - Batsic Reference Model," Intemational Standards Organization, Crenevat. 1984.

|Lyy2| J. Bryan Lyles and Danial C'. Swinchatr. "The Energing Gigahit Environment and the Role of Local ATM." IEEE Communications matga/me, pp. 52-58. April 1992.

|Ma78| James Martin. "Communications Sattellite Systems." Prentice Hall. Englewend Cliffs, N.J., 1978.

|Rat') M. Niel Ransom and Dan R. Spats. "Applications of Public Gigahil Networks," IEEE Network Magavinte vol. 6. no. 2. pr. 311-40. March 1902.

$|S(X)|$ Leconard Stans, M. Bencoe, ('D). Brown. S.M. Kelly, L.G. Pierson. and C. Schaldarch, "Network Archiceture Functional Design Version 2.1)," Sandia National Lahoratories Report, SAND8X-2416. May loxy.

|TiY21 Nick Testi. "Consolidation Projed Texis." Samdia National Lathoratories Memorandum, March 1992.

|Th8e) K.J. Thurber, "Ciettirge a handle on FDDI," IEEE Data Communications Magavine, pp.28-32, June $19 \times 4$.

|To92| Joseph D). Tomch, "Mirage: A Model for Latency in Communication," Ph.D. disscrtation, University of Pennsylvaniat. Philatelphiat. PA. January 1992. 
Copy to:

MS0360 B. Westwood, 1000

MS0320 P. L. Mattern, 1010

MS0321 E. H. Barsis, 1400

MS1109 J. N. Jortner, 1408

MS1111 W. J. Camp, 1421

MS1109 A. L. Hale, 1424

MS0441 J. H. Biffle, 1425

MS0441 J. F. Mareda, 1425

MS0441 C. J. Pavlakos, 1425

MS(0819 J. M. McGlaun, 1431

MS0841 D. J. McCloskey, 1500

MS()826 T. J. Bartel, 1553

MSO803 D. L. Crawford, 1900

MS9011 R. E. Palmer, 1901

MS()803 R. M. Cahoon, 1902

MS0806 D. C. Jones, 1903

MS0805 A. R. Iacoletti, 1904

MS9()40 G. Gutierrez, 1905

MS0806 R. C. Dougherty, 1906

MS0805 C. D. Brown, 1932

MS0805 W. H. Rahe, 1932

MS9011 P. W. Dean, 1951

MS9011 J. C. Berry, 1951

MS9011 F. T. Bielecki, 1951

MSY011 H. Y. Chen, 1951

MS9011 C. Fang, 1951

MS9011 J. A. Hutchins, 1951

MS9011 R. E. Cline, Jr., 1952

MS0807 M. O. Vahle, 1954

MS0807 A. Breckinridge, 1954

MS0807 J. P.Brenkosh, 1954

MS0807 S. A. Gossage, 1954

MS0807 T. C. Hu, 1954

MS0807 J. H. Maestas, 1954

MS0807 L. Martinez, 1954

MS0807 J. H. Naegle, 1954

MS0807 L. G. Pierson, 1954

MS0807 T. J. Pratt, 1954

MS0807 N. Testi, 1954

MS0807 B. Whittet, 1954

MS0807 E. L. Witzke, 1954/(RESPEC)

MS0807 R. C. Dougherty, Actg., 1955

MS0807 R. L. Adams, 1955

MS0807 I. C. Alexander, 1955

MS0807 J. M. Eldridge (15), 1955
MS0807 S. D. Nelson, 1955

MS0807 M. A. Schaefer, 1! j5

MS0807 L. F. Tolendino, 1955

MS0807 J. P. Sena, 1955-1

MS0807 D. W. Tapscott, 1955-1

MS0811 R. J. Pryor, 1956

MS0811 G. D Machin, 1956

MS0806 W. D. Swartz, 1957

MS0806 M. J. Bensen, 1957

MS0806 D. P. Duggan, 1957

MS0806 R. A. Haynes, 1957

MS0806 S. M. Kelly, 1957

MS0806 J. P. Long, 1957

MS0639 D. M. Darsey, 2864

MS(0656 T. J. Draelos, 9249

MS(1899 Technical Library (5), 7141

MS00619 Technical Publications (1), 7151

MS1119 Document Processing

for DOE/OSTI (10), 7613-2

MS9017 Central Technical Files (1), 8523-2 



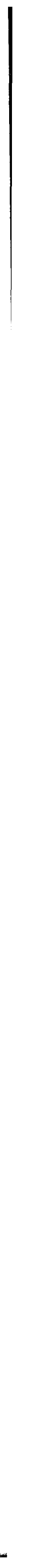

